\title{
SPECTRAL COUPLING OF EFFECTIVE PROPERTIES OF A RANDOM MIXTURE
}

\author{
Elena Cherkaev \\ Department of Mathematics \\ The University of Utah \\ 155 South 1400 East, JWB 233 \\ Salt Lake City, UT, 84112, U.S.A. \\ elena@math.utah.edu
}

Keywords: Spectral measure, coupling, effective properties, Stieltjes function, regularization

\begin{abstract}
The paper deals with the problem of coupling of various effective properties of a random mixture. It is shown that effective properties of a random stationary composite formed of two different materials are coupled through the spectral measure in the Stieltjes representation of the effective properties. This gives an approach to indirect evaluation of the effective thermal or hydraulic conductivity of a random material from known effective complex permittivity of the same mixture. The spectral measure is reconstructed from effective complex permittivity measurements and used to estimate other effective properties of the same material.
\end{abstract}

\section{INTRODUCTION}

Different effective properties of a finely-structured heterogeneous mixture are related or coupled through its microgeometry. The problem of formalizing this coupling of the properties is very important for predicting properties of composite materials in material design, as well as for indirect evaluation of the effective properties, when direct measurements are difficult to make. Implicit accounting for the geometry of the composite was exploited starting from the pioneering work of Prager [1] in deriving coupled bounds on the effective material properties. Coupled or cross-property bounds use measurements of one effective property to improve bounds on other effective properties. The work of Prager was followed by a number of papers by Avellaneda, Berryman, Cherkaev, Gibiansky, Milton, Torquato, 
and others (see references in monographs $[2,3,4]$ ). Various empirical relations or relations derived for specific geometries are used in practice, such as for instance, Kozeny-Carman or Katz-Tompson relations providing an estimate for permeability of a porous material $[5,6]$. Geometric characterization of the medium is often introduced through the "formation factor" $F$ which relates properties of one phase in the mixture to the effective properties of the material: $F=\sigma / \sigma^{*}$, where $\sigma$ is the conductivity of a fluid filling in the porous material, and $\sigma^{*}$ is the effective conductivity.

The present work uses explicit analytic representation of various effective properties of a composite [7] through its geometric structural function associated with the spectral measure $\mu$ in the Stieltjes analytic integral representation of the effective complex permittivity $\eta^{*}$. This analytic integral representation of the effective permittivity $\eta^{*}$ of a mixture of two materials with permittivities $\eta_{1}$ and $\eta_{2}$ was developed by Bergman, Milton, and Golden and Papanicolaou [8, 9, 10, 11] in the course of computing bounds for the effective permittivity of an arbitrary two component mixture. The integral representation gives a function $F(s)$ as an analytic function off $[0,1]$-interval in the complex $s$-plane:

$$
F(s)=1-\frac{\eta^{*}}{\eta_{2}}=\int_{0}^{1} \frac{d \mu(z)}{s-z}, \quad s=\frac{1}{1-\eta_{1} / \eta_{2}}
$$

Here the positive measure $\mu$ is the spectral measure of a self-adjoint operator $\Gamma \chi$, with $\chi$ being the characteristic function of the domain occupied by one material, and

$$
\Gamma=\nabla(-\Delta)^{-1}(\nabla \cdot)
$$

where $-\Delta$ is the Laplacian operator, and $\nabla$ denotes the gradient, so that $(\nabla \cdot)$ is the divergence operator. The spectral function $\mu$ was used to derive microstructural information about the composite $[12,13,14,15]$, to bound the effective permittivity $[16,17,18]$, to appraise the accuracy of the permittivity measurements [19], and to model the effective complex conductivity of geological mixtures [20, 21] or of random resistor networks [22]; it was calculated from reflectivity measurements at different temperatures in $[23]$.

The paper discusses coupling of different properties of a stationary random mixture through the spectral function $\mu$. It is demonstrated in [7] that different properties of a random mixture admit representations similar to (1.1) with the same function $\mu$, and that the effective response of the random medium for a range of different parameters of the applied field determines the function $\mu$. Hence, when computed from measurements of one effective property (say, from measurements of $\eta^{*}$ ), the function $\mu$ can be used to evaluate the effective response of the same medium for 
other applied fields as well. From the computational point of view, the problem of reconstruction of the spectral measure $\mu$ is extremely ill-posed: It is equivalent to the inverse potential problem and is well studied in the mathematical literature (see, for instance, the monograph on inverse source problems [24] and on regularization of ill-posed problems [25]). The last section shows computational results of recovering the spectral function $\mu$ from numerically simulated effective measurements of the complex permittivity of a composite using regularized algorithms developed in $[7,26]$. As an example, the thermal conductivity of St-Peters sandstone is estimated using the reconstructed function $\mu$ [27]. Computed values of the thermal conductivity are in good agreement with measured values in [28].

\section{COUPLING THROUGH THE SPECTRAL FUNCTION}

We assume that the medium in a domain $\mathcal{O}$ is a fine scale mixture of two materials with the values of the complex permittivities $\epsilon_{j}$ and the thermal conductivities $\gamma_{j}$ in regions $\mathcal{O}_{j}, j=1,2$, with $\mathcal{O}=\mathcal{O}_{1} \cup \mathcal{O}_{2}$. Let $\chi$ be the characteristic function of the region $\mathcal{O}_{1}$ occupied by the first material for a realization $\eta \in \Omega$, where $\Omega$ is the set of all realizations of the random medium,

$$
\chi(x, \eta)=\left\{\begin{array}{l}
1, x \in \mathcal{O}_{1} \\
0, \text { otherwise }
\end{array}\right.
$$

We assume that the characteristic size of the inhomogeneities is microscopic in comparison with the size of the region $\mathcal{O}$, hence $\chi$ is a finely oscillating function.

Suppose that two different fields are applied in this random medium, the electric field, $E_{\eta}$, and the temperature gradient field, $E_{\gamma}$. The complex permittivity of the medium is modeled by a (spatially) stationary random field $\epsilon(x, \eta), x \in R^{d}$ and $\eta \in \Omega, \epsilon(x, \eta)=\epsilon_{1} \chi(x, \eta)+\epsilon_{2}(1-\chi(x, \eta))$. Similarly, the thermal conductivity is $\gamma(x, \eta)=\gamma_{1} \chi(x, \eta)+\gamma_{2}(1-\chi(x, \eta))$. Since the stationary fields are governed by similar equations, we will use the notation $\sigma$ to describe both properties. The stationary random fields $E_{\sigma}(x, \eta)$ and $J_{\sigma}(x, \eta)$ are related by $J_{\sigma}(x, \eta)=\sigma(x, \eta) E_{\sigma}(x, \eta)$ and satisfy the equations

$$
\nabla \cdot J_{\sigma}=0, \quad \nabla \times E_{\sigma}=0, \quad\left\langle E_{\sigma}(x, \eta)\right\rangle=e_{k}, \quad \sigma=\eta, \gamma .
$$

Here $e_{k}$ is a unit vector in the $k^{t h}$ direction, for some $k=1, \ldots, d$, and $\langle\cdot\rangle$ means ensemble average over $\Omega$ or spatial average over all of $\mathbf{R}^{d}$. The effective tensor $\sigma^{*}$ is defined as a coefficient of proportionality between the averaged fields: $\left\langle J_{\sigma}\right\rangle=\sigma^{*}\left\langle E_{\sigma}\right\rangle$. Hence the effective property tensors $\eta^{*}$ 
and $\gamma^{*}$ are such that

$$
\left\langle J_{\eta}\right\rangle=\eta^{*}\left\langle E_{\eta}\right\rangle \quad \text { and } \quad\left\langle J_{\gamma}\right\rangle=\gamma^{*}\left\langle E_{\gamma}\right\rangle
$$

We notice that both problems are coupled through the same function $\chi$ :

$$
\nabla \cdot\left(\eta_{1} \chi(x, \eta)+\eta_{2}(1-\chi(x, \eta))\right) E_{\eta}=0, \quad \eta^{*}=\left\langle\eta E_{\eta}\right\rangle
$$

and

$$
\nabla \cdot\left(\gamma_{1} \chi(x, \eta)+\gamma_{2}(1-\chi(x, \eta))\right) E_{\gamma}=0 \quad \gamma^{*}=\left\langle\gamma E_{\gamma}\right\rangle
$$

Suppose that one of the effective properties, $\eta^{*}$ (see 2.4), can be measured. The problem is to find the other effective property $\gamma^{*}$ using (2.5). We consider here isotropic mixtures and focus on one diagonal coefficient $\eta^{*}=$ $\eta_{k k}^{*}$ and $\gamma^{*}=\gamma_{k k}^{*}$.

Let the complex permittivities $\eta_{i}, i=1,2$, depend on a parameter $p$ that can be varied, with the microstructure remaining the same. This means that variation of the parameter $p$ does not change the function $\chi$. The temperature dependent complex permittivity of sea ice provides an example of a medium which does not fall into this consideration, since induced by temperature variations, melting changes the structure of brine pockets.

Theorem. Assuming the known properties $\eta_{i}=\eta_{i}(p), i=1$ or $i=$ 2 , of materials in a stationary random mixture depend on a parameter $p$, measurements of the effective complex permittivity $\eta^{*}(p)(2.4)$ in an interval $p \in\left(p_{1}, p_{2}\right)$ uniquely determine the effective thermal conductivity $\gamma^{*}(2.5)$ of the mixture for given values of the components $\gamma_{i}, i=1,2$.

The key observation is that the effective properties $\eta^{*}$ and $\gamma^{*}$ are coupled through the function $\mu$ in their integral representation [7]:

$$
\begin{array}{cc}
\eta^{*}(s)=\epsilon_{2}-\epsilon_{2} \int_{0}^{1} \frac{d \mu(z)}{s-z}, & s=\frac{1}{1-\epsilon_{1} / \epsilon_{2}} \\
\gamma^{*}\left(s^{\prime}\right)=\gamma_{2}-\gamma_{2} \int_{0}^{1} \frac{d \mu(z)}{s^{\prime}-z}, & s^{\prime}=\frac{1}{1-\gamma_{1} / \gamma_{2}}
\end{array}
$$

To demonstrate this, we consider a model problem for a random mixture of two materials with conductivity $\sigma_{1}=h$ and $\sigma_{2}=1$, and derive the spectral integral representation for the effective property following [11]. The conductivity $\sigma(x, \eta)$ of the mixture is $\sigma(x, \eta)=h \chi(x, \eta)+(1-\chi(x, \eta))$. The field $E$ satisfies

$$
\nabla \cdot(h \chi+1-\chi) E=0
$$


Introducing $s=1 /(1-h)$, the last expression can be written as

$$
\nabla \cdot \chi E=s \nabla \cdot E, \quad s=\frac{1}{1-h}
$$

Let $\nabla \varphi$ be a perturbation of the constant field $e_{k}$, so that $E=e_{k}+\nabla \varphi$. Then,

$$
\nabla \cdot \chi\left(\nabla \varphi+e_{k}\right)=s \Delta \varphi
$$

Applying $(-\Delta)^{-1}$ to both sides of this equation, then taking gradient, and introducing the operator $\Gamma$ as in $(1.2), \Gamma=\nabla(-\Delta)^{-1}(\nabla \cdot)$, we can express $E$ as a function of $\Gamma \chi$,

$$
E=s(s I+\Gamma \chi)^{-1} e_{k} .
$$

The spectral resolution of $\Gamma \chi$ with the projection valued measure $Q$ results in the representation

$$
E(s)=\int_{0}^{1} \frac{s}{s-z} d Q(z) e_{k}
$$

The integral represention for the function $F(s)$ is obtained using (2.12). Indeed,

$$
F(s)=1-\sigma^{*}(s)=\left\langle s^{-1} \chi E, e_{k}\right\rangle
$$

and hence,

$$
F(s)=\left\langle\chi(s I+\Gamma \chi)^{-1} e_{k}, e_{k}\right\rangle=\int_{0}^{1} \frac{\left\langle\chi d Q(z) e_{k}, e_{k}\right\rangle}{s-z}
$$

If a function $\mu$ is a positive function of bounded variation, corresponding to the spectral measure $Q, d \mu(z)=\left\langle\chi d Q(z) e_{k}, e_{k}\right\rangle$, then

$$
F(s)=\int_{0}^{1} \frac{d \mu(z)}{s-z}
$$

Substituting now in this model problem the value of $h$ as $h=\eta_{1} / \eta_{2}$ and $h=\gamma_{1} / \gamma_{2}$, we end up with representations (2.6) and (2.7).

It is shown in [7] that the spectral function $\mu$ in the Stieltjes integral representation can be uniquely reconstructed if measurements of the effective permittivity of the mixture $\eta^{*}$ are available on an arc in a complex plane. Provided the properties of the constituents are dependent on a parameter $p$, variation of $p$ in an interval $p \in\left(p_{1}, p_{2}\right)$ gives the required set of data.

If the properties of the constituents depend on the frequency of the applied electromagnetic field, frequency can be taken as such a parameter 
which variation does not change the structure of the mixture. The spectral measure $\mu$ is determined by the structure of the mixture, hence, it is quite natural that it is the same for different effective properties. Having reconstructed this function from one set of data, we can use it to compute other effective properties such as thermal or hydraulic conductivity of the same mixture. Practically, if the function $\mu$ is known from the measurements of $\eta^{*}$, evaluation of the effective thermal conductivity $\gamma^{*}$ reduces to a calculation of the integral in (2.7).

Similarly, we could consider other stationary problems for:

- diffusion coefficient with concentration gradient and mass flux

- hydraulic conductivity with pressure gradient and fluid velocity

- magnetic permeability with magnetic field and magnetic induction

\section{REGULARIZATION}

The problem of reconstruction of the spectral measure $\mu$ can be reduced to an inverse potential problem. It is shown that the function $F(s)$ admits a representation as a logarithmic potential of the measure $\mu[7]$

$$
F(s)=\frac{\nabla}{\nabla s} \int \ln |s-z| d \mu(z), \quad \nabla / \nabla s=(\nabla / \nabla x-i \nabla / \nabla y)
$$

The reconstruction problem for the logarithmic potential is extremely illposed and requires regularization to develop a stable numerical algorithm. The potential function $u$ is a solution to the Poisson equation $-\Delta u=$ $\psi, \quad \operatorname{supp}(\psi) \subset \Omega$, where $\psi$ is the density of the mass distribution in $\Omega$. Solution of the problem is given by the Newtonian potential with $d \mu(z)=$ $\psi d z, \quad z \in \Omega$. The inverse problem is to find $\psi$ given values of $\partial u / \partial n$, or $\nabla u$.

Let $A$ be an operator in (3.1) mapping the set of measures $\mathcal{M}[0,1]$ on the unit interval onto the set of complex potentials defined on a curve $\mathcal{C}$ : $\zeta(s)=0$ :

$$
A \mu(s)=f(s)+i g(s)=\frac{\nabla}{\nabla s} \int_{0}^{1} \ln |s-z| d \mu(z), \quad s \in \mathcal{C} .
$$

To construct the solution we formulate the minimization problem:

$$
\|A \mu-F\| \rightarrow \min _{\mu \in \mathcal{M}}
$$

where $\|\cdot\|$ is the $L^{2}(\mathcal{C})$-norm, $F$ is the function of the measured data, $F(s)=1-\eta^{*}(s) / \eta_{2}, s \in \mathcal{C}$. The solution of the problem does not continuously depend on the data: Unboundness of the operator $A^{-1}$ leads 
to arbitrarily large variations in the solution, and the problem requires a regularization technique.

A regularization algorithm developed in [7] is based on constrained minimization: It introduces a stabilization functional $J(\mu)$ which constrains the set of minimizers. As a result, the solution depends continuously on the input data. Instead of minimizing (3.3) over all functions in $\mathcal{M}$, minimization is performed over a convex subset of functions which satisfy $J(\mu) \leq \beta$, for some scalar $\beta>0$. The functional $J(\mu)$ was chosen as a quadratic stabilization functional and a total variation functional.

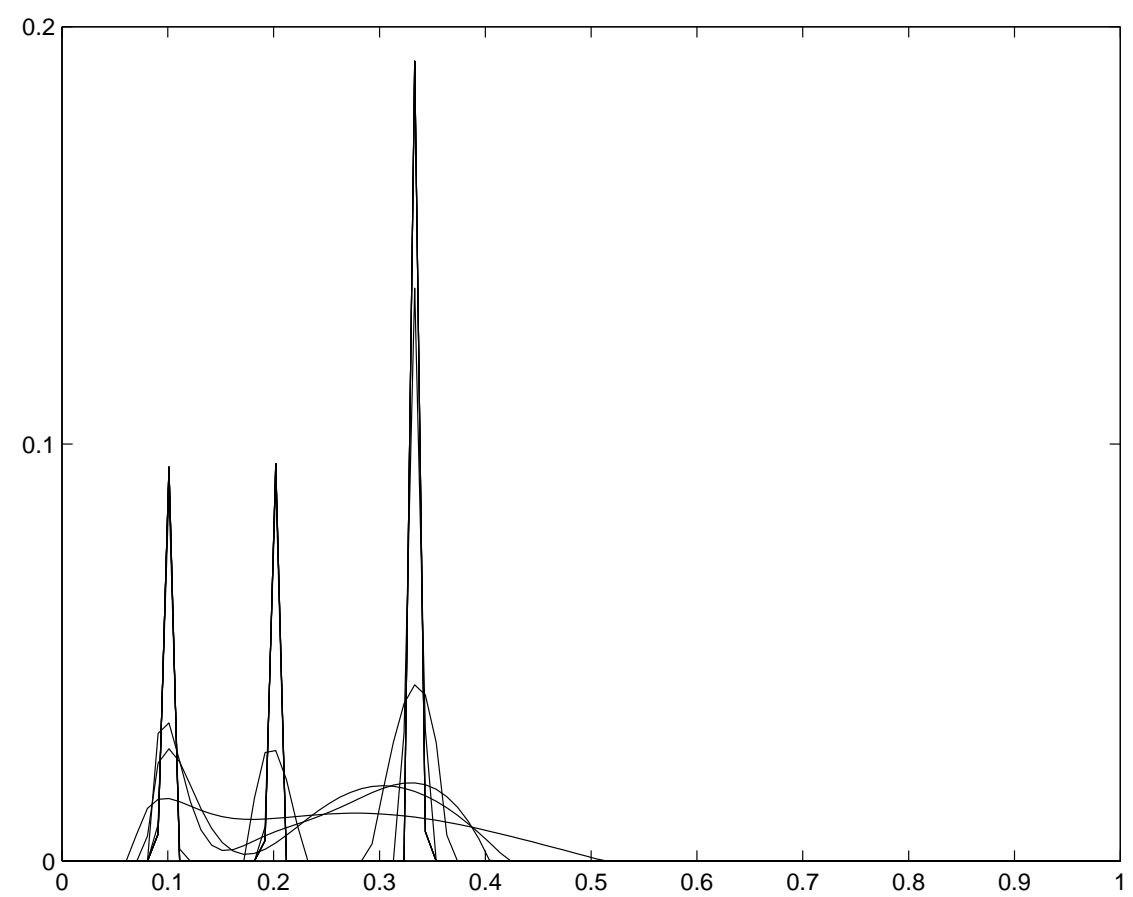

Figure 1 Reconstruction of a three-resonance structural function. Smooth functions are reconstructed using Tikhonov regularization with different regularization parameters $\alpha$. The three delta-functions are recovered (almost identically to the true solution) using non-negatively constrained minimization [26].

The advantage of using a quadratic stabilization functional $J(\mu)=$ $\|L \mu\|^{2}$, is the linearity of the corresponding Euler equation resulting in efficiency of the numerical schemes: $\mu_{\alpha}=\left(A^{*} A+\alpha L^{*} L\right)^{-1} A^{*} F^{\delta}$. However, the reconstructed solution necessarily possesses a certain smoothness. The alternative nonquadratic stabilization functional imposes constraint on the variation of the solution in the domain. The total variation penalization, as well as the regulariization based on the non-negativity constraint [26], does 
not impose smoothness on the solution, which permits recovering blocky and contrast structures.

Accurate reconstruction of the function $\mu$ is especially important when the initial materials' constants are in the vicinity of the spectral interval, say as in the case of percolation. In [26], we assume that the medium formed as a mixture of sandgrains and water, has three resonances. The corresponding structural function $\mu$ is shown in Figure 1 as a sum of three delta functions. Numerically simulated multi-frequency values of the imaginary part of the effective complex permittivity were used to recover the structural function $\mu$. The functions $\mu$ reconstructed using Tikhonov and non-negatively constrained regularization, are also shown in the same figure. While the Tikhonov regularization gives oversmoothed curves, the non-negatively constrained solution practically exactly reconstructs the true function: The difference is indistinguishable on the given scale.

\section{COMPARISON WITH EXPERIMENT}

For appraisal of the suggested indirect method of computation of the effective properties of the composite from known effective complex permittivity, the approach was applied in [27] to the data for St-Peters sandstone, which is composed of sandgrains and has $11 \%$ porosity. Assuming porous space filled with water, with known dependence of $\eta_{\text {water }}$ on frequency, measurements of the effective $\eta^{*}(\odot)$ were simulated using the MaxwellGarnett model. These simulated data of $\eta^{*}$ were used to compute the

Table 1 Thermal conductivity of St-Peters sandstone

\begin{tabular}{ccc}
\hline & Wet sandstone & Dry sandstone \\
\hline Measured & 6.36 & 3.56 \\
\hline Computed & 6.63 & 3.55 \\
\hline
\end{tabular}

spectral function $\mu$, which was used then to calculate the thermal conductivity of the rock. Computed and measured data for wet (sandgrains and $11 \%$ of water) and dry (sandgrains and $11 \%$ of air) sandstone are summarized in Table 1. Values of the thermal conductivity computed using the suggested algorithm are in good agreement with experimental data taken for comparison from [28]. 


\section{CONCLUSION}

It is shown that various effective properties of a stationary random mixture are coupled through the spectral function. The spectral function can be found from measurements of effective complex permittivity and used to calculate other effective properties. The approach is applicable to porous media, biological materials, artificial composites, and other heterogeneous materials in which the scale of the microstructure is much smaller than the wavelength of the electromagnetic signal.

\section{References}

[1] Prager, S (1969) Improved variational bounds on some bulk properties of a two-phase random medium, J. Chem. Phys. 50(10), 4305-4312.

[2] Cherkaev, A (2000) Variational methods for structural optimization, New York: Springer-Verlag.

[3] Milton, G W (2002) Theory of composites, Cambridge Press.

[4] Torquato, S (2002) Random heterogeneous materials. Microstructure and macroscopic properties, New York: Springer-Verlag.

[5] Avellaneda, M and Torquato, S (1991) Rigorous link between fluid permeability, electrical conductivity, and relaxation times, Phys. Fluids A 3(11), 2529-2540.

[6] Berryman, J G and Blair, S C (1987) Kozeny-Carman relations and image processing methods for estimating Darcy's constant, J. Appl. Phys. 62, 2221-2228.

[7] Cherkaev, E (2001) Inverse homogenization for evaluation of effective properties of a mixture, Inverse Problems 17, 1203-1218.

[8] Bergman, D J (1978) The dielectric constant of a composite material A problem in classical physics, Phys. Rep. C 43, 377-407.

[9] Bergman, D J (1980) Exactly solvable microscopic geometries and rigorous bounds for the complex dielectric constant of a two-component composite material, Phys. Rev. Lett. 44, 1285.

[10] Milton, G W (1980) Bounds on the complex dielectric constant of a composite material, Appl. Phys. Lett. 37(3), 300-302.

[11] Golden, K and Papanicolaou, G (1983) Bounds on effective parameters of heterogeneous media by analytic continuation, Comm. Math. Phys. 90, 473-491.

[12] McPhedran, R C, McKenzie, D R and Milton, G W (1982) Extraction of structural information from measured transport properties of composites, Appl. Phys. A 29, 19-27. 
[13] Gajdardziska-Josifovska, M, McPhedran, R C, McKenzie, D R and Collins, R E (1989) Silver-magnesium fluoride cermet films. 2: Optical and electrical properties, Applied Optics 28(14), 2744-2753.

[14] McPhedran, R C and Milton, G W (1990) Inverse transport problems for composite media, Mat. Res. Soc. Symp. Proc. 195, 257-274.

[15] Cherkaeva, E and Golden, K M (1998) Inverse bounds for microstructural parameters of a composite media derived from complex permittivity measurements, Waves in Random Media 8, 437-450.

[16] Milton, G W (1981) Bounds on the complex permittivity of a two component composite material, J. Appl. Phys. 52(8), 5286-5293.

[17] Bergman, D J (1982) Rigorous bounds for the dielectric constant of a two-component composite, Ann. Phys. 138, 78.

[18] Bergman, D J (1993) Hierarchies of Stieltjes functions and their application to the calculation of bounds for the dielectric constant, SIAM, J. Appl. Math. 53(4), 915-930.

[19] Eyre, D, Milton, G W and Mantese, J V (1997) Finite frequency range Kramers Kronig relations: Bounds on the dispersion, Phys. Rev. Lett. 79, 3062-3064.

[20] Cherkaeva, E and Tripp, A C (1996) Bounds on porosity for dielectric logging, ECMI96, 9th Conference of the European Consortium for Mathematics in Industry, Denmark, 304-306.

[21] Tripp, A C, Cherkaeva, E and Hulen, J (1998) Bounds on the complex conductivity of geophysical mixtures, Geophysical Prospecting 46(6), 589-601.

[22] Day, A R and Thorpe, M F (1996) The spectral function of random resistor networks, J. Phys. Condens. Matter 8, 4389-4409.

[23] Day, A R, Thorpe, M F, Grant, A R and Sievers, A J (2000) The spectral function of a composite from reflectance data, Physica $\mathrm{B}, \mathbf{2 7 9}$, $17-20$.

[24] Isakov, V (1990) Inverse source problems, Providence: AMS, Math. Surveys and Monographs, 34.

[25] Tikhonov, A N and Arsenin, V Y (1977) Solutions of ill-posed problems, New York: Willey.

[26] Cherkaev, E , Hansen, P C and Berglund, A (2002) Regularization using non-negativity constraints.

[27] Cherkaev, E, Zhang, D and Tripp, A C (2002) Remote electromagnetic sensing of the thermal conductivity in the subsurface.

[28] Woodside, W and Messmer, J H (1961) Thermal conductivity of porous media II: Consolidated rocks, J Appl. Phys. 43(9), 1699-1706. 